\title{
Fatores determinantes da participação em iniciativas de Crowdsourcing
}

Ivan Roberto Ferraz

Doutor em Administração pela Universidade de São Paulo; Mestre em Administração pela Pontifícia Universidade Católica de São Paulo, especialista em Análise de Dados e Data Mining pela Fundação Instituto de Administração e bacharel em Administração pela Universidade Presbiteriana Mackenzie. É professor em cursos de graduação e pós-graduação. São Paulo, Brasil ivanferraz@hotmail.com

Maria Aparecida Gouvêa

Graduação em Estatística e em Letras; Mestrado em Estatística; Doutora em Administração; LivreDocência em Administração e de Aperfeiçoamento na Metodologia do Estudo de Caso na Harvard University (2003). Professora Titular no Departamento de Administração da FEA/USP e Pesquisadora Bolsista de Produtividade (1D/CNPq). São Paulo, Brasil magouvea@usp.br

Iná Futino Barreto

Doutora em administração de Empresas pela FEA-USP; Mestre em Administração de Empresas pela FEA-USP. Graduação em Comunicação Social pela Escola Superior de Administração Marketing e Comunicação. Docente na Fundação Escola de Comércio Álvares Penteado (FECAP) e Escola Superior de Propaganda e Marketing (ESPM), São Paulo, Brasil inafb@uol.com.br

Editor Científico: José Edson Lara Organização Comitê Científico

Double Blind Review pelo SEER/OJS

Recebido em 22.07.2016

Aprovado em 14.03.2017

Este trabalho foi licenciado com uma Licença Creative Commons - Atribuição - Não Comercial 3.0 Brasil 


\title{
Resumo
}

Crowdsourcing é um tema emergente, potencializado pela revolução tecnológica e de comunicações. Este trabalho buscou identificar fatores que influenciam a participação em crowdsourcing e verificar se a relevância destes fatores muda conforme a modalidade do projeto. A partir de uma extensa revisão da literatura sobre o tema, foram selecionados 36 trabalhos que identificaram algum fator relevante. A categorização e análise dos resultados destes trabalhos levaram à identificação de 13 fatores: prazer, recompensa, autodesenvolvimento, reputação e carreira, social, altruísmo, simplicidade, significado da tarefa, autonomia, possui habilidade, confiabilidade, feedback e interesse. Destes, "Prazer", "Recompensa", "Autodesenvolvimento" e "Reputação/Carreira" se mostraram os mais importantes. Também foram encontradas evidências de que a importância relativa de motivações intrínsecas ou extrínsecas varia conforme a modalidade de crowdsourcing. Esses resultados podem ajudar as organizações a criarem projetos que tenham maior chance de sucesso.

Palavras Chave: Crowdsourcing, motivadores, participação.

\section{Determinants factors of participation in Crowdsourcing initiatives}

\begin{abstract}
Crowdsourcing is an emerging theme, powered by the technological revolution and communications. This study aimed to identify factors that influence participation in crowdsourcing and verify if the relevance of these factors are the same for the different types of crowdsourcing projects. From an extensive review of literature on the subject, 36 studies that identified a relevant factor for the participation were selected. The categorization and analysis of the results of these 36 studies led to the identification of 13 factors: enjoyment, reward, self-development, reputation and career, social, altruism, simplicity, "meaningfulness" of the task, autonomy, selfeficacy, reliability, feedback and interest. Of these, "Enjoyment," "Reward", "Selfdevelopment" and "Reputation / Career" were the most important. The results also show that the relative importance of intrinsic or extrinsic motivations varies according to the type of the crowdsourcing project. These results may help organizations in the conception of projects with greater chance of success.
\end{abstract}

Keywords: Crowdsourcing, motivators, participation.

\section{Fatores determinantes de la participación en iniciativas de Crowdsourcing}

\section{Resumen}

El crowdsourcing es un tema emergente, impulsado por la revolución tecnológica y de las comunicaciones. Este estudio tuvo como objetivo identificar los factores que influyen en la participación en el crowdsourcing y comprobar si la relevancia de estos factores son los mismos para los diferentes tipos de proyectos de crowdsourcing. A partir de una extensa revisión de la literatura sobre el tema, se seleccionaron 36

$@ \otimes$ Revista Gestão \& Tecnologia, Pedro Leopoldo, v. 17, n. 1, p. 107-129, jan./abr. 2017108 
estudios que identificaron un factor relevante. La categorización y análisis de los resultados de estos 36 estudios llevaron a la identificación de los 13 factores: el placer, de recompensa, de auto-desarrollo, reputación y su carrera, social, generosidad, sencillez, de significación tarea, autonomía, tiene la capacidad, la fiabilidad, la retroalimentación y el interés. De estos, "placer", "recompensa", "autodesarrollo" y "la reputación / carrera" fueron los más importantes. También existe evidencia de que la importancia relativa de las motivaciones intrínsecos o extrínsecos varía según el tipo de crowdsourcing. Estos resultados pueden ayudar a las organizaciones a crear proyectos que tienen una mayor probabilidad de éxito.

Palabras clave: Crowdsourcing, motivadores, la participación

\section{Introdução}

$O$ avanço tecnológico, intensificado a partir do final do século $X X$, tem revolucionado o acesso à informação e os processos de comunicação. Uma enorme parcela do conhecimento humano já está disponível em formato digital, e tecnologias como a Internet têm contribuído para a democratização do acesso a esse conteúdo e permitido a formação de redes de comunicação e colaboração que não seriam possíveis há apenas alguns anos. Além disso, o custo dessas tecnologias é cada vez mais baixo e, a cada ano, uma parcela maior da população tem acesso a computadores, smartphones, tablets e a conexões de Internet de alta velocidade (CGI.BR, 2016).

Muitas são as mudanças na sociedade provocadas por essa revolução digital e de comunicações (Castells, 1999; Toffler, 1980). Surgem novas dinâmicas de produção, trabalho, ensino, relacionamento; enfim, várias esferas da vida em sociedade estão sofrendo transformações profundas em um curto período de tempo e a humanidade ainda está aprendendo a lidar com as consequências desse processo. Esse avanço tecnológico, em especial o advento da Internet e da World Wide Web (WEB), possibilita maior exploração das potencialidades da coletividade, ou seja, do trabalho ou colaboração de uma grande rede de indivíduos.

Antes da revolução digital das últimas décadas, o processo de agregação de informações dispersas em grandes grupos, quando viável, era penoso e custoso. Trabalhos em colaboração demandavam interações presenciais ou por meio de sistemas de comunicação menos eficientes. Todavia, as novas tecnologias que surgiram no final do século XX mudaram essa situação. A partir do momento em que 
a Internet tornou-se acessível a um número maior de pessoas, naturalmente surgiram inúmeras iniciativas que tiram proveito do poder dos grandes grupos. É possível citar inúmeros exemplos dessas iniciativas, como a Wikipédia, o desenvolvimento de software livre ou o Google.

A velocidade com que ideias novas surgem, são implementadas, replicadas e aperfeiçoadas é tão rápida que é difícil entender e explicar tudo o que está acontecendo. Inteligência Coletiva, Sabedoria das Multidões, WEB 2.0, Crowdsourcing, Crowdfunding, CrowdStorm, etc., são todos termos novos para explicar ou classificar essas iniciativas que visam a explorar o poder da coletividade, potencializado pelas novas tecnologias.

Este artigo discute especialmente o Crowdsourcing, termo que apareceu pela primeira vez no ano de 2006 em um artigo da revista Wired (Howe, 2006) e diz respeito a um modelo de produção ou resolução de problemas que ocorre de forma online e distribuída. As organizações perceberam que, da mesma forma que conquistam vantagens competitivas terceirizando produtos e serviços para outras empresas, podem obter vantagens terceirizando tarefas para a coletividade.

Segundo Howe (2006), crowdsourcing representa a ação de uma organização que seleciona uma atividade antes realizada por funcionários e a terceiriza para uma rede indefinida de pessoas, sob a forma de um convite aberto. A respectiva atividade pode ser realizada em colaboração, mas também pode ser realizada por indivíduos trabalhando sozinhos. É fundamental, entretanto, o uso do formato de convite aberto e a grande rede de potenciais colaboradores.

Diversos tipos de trabalhos podem ser terceirizados dessa forma, desde tarefas simples até outras extremamente complicadas e que exigem competências específicas. Por exemplo, no site Mechanical Turk da Amazon, trabalhadores do mundo inteiro oferecem sua mão de obra para a realização de tarefas simples, como, por exemplo, a categorização de imagens ou transcrição de áudio, em troca de pequenas remunerações (Kaufmann, Schulze, \& Veit, 2011). Já no site InnoCentive, lançado em 2001 com financiamento da gigante farmacêutica Eli Lilly (Howe, 2006), grandes empresas oferecem recompensas generosas em troca de soluções para problemas complexos de pesquisa e desenvolvimento. Apesar de qualquer pessoa interessada poder propor soluções, independentemente de sua qualificação profissional, muitas ideias inovadoras e de qualidade são recebidas pelas empresas. 
Tanto Mechanical Turk quanto InnoCentive são exemplos de crowdsourcing pois há um convite aberto (feito por meio da internet), para que uma rede grande de pessoas possa colaborar e, assim, terceirizar uma atividade que teria que ser desenvolvida internamente. Por exemplo, um pesquisador pode, em vez de ele mesmo transcrever suas entrevistas, incluir uma oferta no Mechanical Turk. O site, então, apresentará a proposta a uma rede enorme de possíveis colabores e um deles poderá realizar a transcrição por um preço adequado e qualidade satisfatória.

A aplicabilidade do crowdsourcing não está apenas em seu potencial de terceirizar atividades, mas sim no quanto o processo de terceirização se torna mais rápido, barato e eficiente por meio da conexão de uma grande quantidade de possíveis colaboradores. O crowdsourcing também pode ser utilizado para ajudar pequenas empresas a encontrarem fornecedores mais rápidos e baratos para funções que não sejam centrais (como desenvolvimento de logotipos, por exemplo); pode ajudar empresários a desenvolver produtos mais adequados a seus consumidores (pois sugestões de produtos e avaliação das sugestões podem ocorrer por meio de crowdsourcing). Inúmeras são as possíveis aplicações práticas que se apresentam nesse tipo de atividade.

Muito por causa de sua grande aplicabilidade, o crowdsourcing é um tema emergente. Entretanto, como é baseado na ideia de que determinada atividade ou tarefa pode ser realizada por um grande grupo de indivíduos conectados em rede, o sucesso de qualquer iniciativa de crowdsourcing depende da participação de pessoas engajadas e dispostas a ceder parte de seu tempo livre, contribuindo com seus conhecimentos e habilidades. Surge, então, a pergunta: como fazer as pessoas participarem de um projeto de crowdsourcing? Essa é a questão de pesquisa que norteia o presente estudo.

Apesar de o modelo já ter sido aplicado com sucesso em diversas iniciativas e nos mais diferentes contextos, a questão de como atrair a participação das pessoas ainda permanece sem uma resposta satisfatória (Brabham, 2008a). Conseguir um número adequado de participantes é fundamental para o sucesso de qualquer projeto que vise a explorar o poder dos grandes grupos. Assim, este trabalho tem dois objetivos:

- Objetivo 1: identificar os fatores que influenciam a participação das pessoas em iniciativas de crowdsourcing. 
- Objetivo 2: verificar se a relevância dos fatores identificados varia em função de diferentes modalidades de crowdsourcing.

\section{Referencial Teórico}

O limite do que é ou deixa de ser uma prática de crowdsourcing nem sempre é claro, devido à diversidade de práticas que o termo pode englobar. Estellés-Arolas e Guevara (2012) compilaram diferentes definições para o termo encontradas na literatura científica e extraíram os elementos comuns, de modo a estabelecer as características básicas de qualquer iniciativa de crowdsourcing. Com base nessas características, os autores propõem a seguinte definição que também foi adotada neste estudo:

Crowdsourcing é um tipo de atividade participativa online em que um indivíduo, uma instituição, uma organização sem fins lucrativos, ou uma empresa propõe, por meio de um convite aberto flexível, o compromisso voluntário de uma tarefa a um grupo heterogêneo de indivíduos com conhecimentos diversos e em número variado. $\mathrm{O}$ empreendimento da tarefa, de complexidade e modulação variáveis, no qual a multidão deve participar contribuindo com seu trabalho, dinheiro, conhecimento e/ou experiência, sempre implica em benefício mútuo. O usuário receberá a satisfação de um determinado tipo de necessidade, seja ela econômica, reconhecimento social, autoestima, ou o desenvolvimento de competências individuais, enquanto o patrocinador (crowdsourcer) irá obter e utilizar a seu favor o que o usuário fornecer, sendo que a maneira de utilização dependerá do tipo de atividade específica (Estellés-Arolas \& Guevara, 2012, p. 197).

Esta definição estabelece oito características que servem como critério para identificar se determinada atividade pode ou não ser classificada como uma iniciativa de crowdsourcing. De acordo com Estellés-Arolas e Guevara (2012), essas características são:

(a) há uma multidão claramente definida;

(b) há uma tarefa com objetivos claros;

(c) a recompensa recebida pela multidão é clara;

(d) o patrocinador (crowdsourcer) é facilmente identificado;

(e) a compensação a ser recebida pelo crowdsourcer está claramente definida;

(f) é um processo atribuído online e do tipo participativo;

(g) é utilizado um convite aberto de extensão variável;

(h) é utilizada a internet. 
Uma atividade que possua todas as características supracitadas pode, sem dúvida, ser considerada uma ação de crowdsourcing. Este é o caso, por exemplo, da Threadless, uma empresa online que comercializa camisetas cujo design é criado e selecionado pelos próprios consumidores. No Threadless há uma multidão definida (característica a) que são, exatamente, os criadores e aqueles que selecionam os melhores designs; há um objetivo claro (característica b): criar e selecionar designs de camisetas; há uma recompensa clara para a multidão (característica $\mathrm{c}$ ), pois aqueles que tem seus designs selecionados recebem prêmios; o patrocinador é o próprio site, que organiza todo o processo, sendo portanto facilmente identificado (característica d); a recompensa do patrocinador também é clara (característica e), pois o site reduz seus custos principalmente por não precisar manter uma equipe própria de designers; o processo funciona por meio da colaboração do público (característica f); todos podem participar livremente (característica g); e, por fim, o site funciona por meio da internet (característica g).

Quanto menos dessas características a atividade possuir, menos adequada é a utilização do termo. O Youtube, por exemplo, apesar de ser uma plataforma de vídeo na internet onde o conteúdo é criado pelos próprios usuários, não pode ser considerado crowdsourcing, pois não atende a maior parte das características elencadas. Apesar de haver uma multidão envolvida (característica a), não há nenhum objetivo ou tarefa comum indicada (característica b). Também não há recompensas claras (característica c). Não há, por exemplo, nenhum tipo de prêmio estabelecido para vídeos com maior quantidade de visualizações e nem nada do gênero. Como não há recompensas ou apresentação de objetivos e tarefas a serem alcançados, não há um patrocinador, ou aquele que estipule as atividades (característica d). Diferente do site Threadless, que pede o envio de designs e organiza a votação neles para seleção dos melhores, o YouTube não solicita nenhum tipo de ação e nem define regras para avaliação ou seleção de submissões. Como não há patrocinador, não existe indicação de o que o patrocinador recebe (característica e). Apesar de contar com um público gigantesco, as atividades do YouTube não são colaborativas (característica f). Cada canal inclui seus vídeos já finalizados que são, então, assistidos por aqueles que se interessem, mas não há 
construção conjunta. Como não há tarefa proposta, não há convite aberto (característica g) para essa proposta.

Na Tabela 1, extraída de Estellés-Arolas e Guevara (2012), analisam-se outras iniciativas, além do Threadless e Youtube, a fim de verificar se elas se encaixam ou não na definição proposta. As iniciativas analisadas são: Wikipédia (uma enciclopédia colaborativa online), InnoCentive (uma plataforma online em que são oferecidos prêmios em dinheiro para a resolução de problemas), Amazon Mechanical Turk (uma plataforma em que os crowdsourcers podem propor a realização de tarefas em troca de pequenas remunerações), ModCloth (uma loja de roupas na internet que permite que seus usuários opinem e votem em designs de roupas antes de sua venda), Lánzanos (um website espanhol em que as pessoas investem em projetos e são recompensadas por seu apoio), Delicious (um sistema de bookmarking social), Fiat Mio (uma iniciativa da empresa Fiat na qual um carro foi desenvolvido a partir de sugestões dos consumidores), iStockphoto (uma plataforma de venda de imagens na internet) e o Flickr (uma plataforma online que permite o envio e a classificação de fotografias).

\section{Tabela 1}

Verificação da Definição de Crowdsourcing

\begin{tabular}{c|c|c|c|c|c|c|c|c}
\hline & a & b & C & d & e & f & g & $\mathbf{h}$ \\
\hline Wikipédia & + & + & + & - & - & + & - & + \\
\hline InnoCentive & + & + & + & + & + & + & + & + \\
\hline Threadless & + & + & + & + & + & + & + & + \\
\hline Amazon Mechanical Turk & + & + & + & + & + & + & + & + \\
\hline ModCloth & + & + & + & + & + & + & + & + \\
\hline YouTube & + & - & - & - & - & - & - & + \\
\hline Lánzanos & + & + & + & + & + & + & + & + \\
\hline Delicious & + & - & - & - & - & - & - & + \\
\hline Fiat Mio & + & + & + & + & + & + & + & + \\
\hline iStockphoto & + & + & + & + & + & + & + & + \\
\hline Flickr & + & - & - & + & - & - & - & + \\
\hline
\end{tabular}

Fonte: Estellés-Arolas, E., \& González-Ladrón-De-Guevara, F. (2012). Towards an integrated crowdsourcing definition. Journal of Information science, 38(2, p. 197), 189-200.

É importante observar que, pela definição adotada, a atividade de desenvolvimento de software livre (Open Source) não é necessariamente uma atividade de crowdsourcing. Embora seja uma atividade realizada de maneira colaborativa pela internet, não há um convite aberto e o crowdsourcer não pode ser facilmente identificado, de modo que sua recompensa também não está clara. 
Assim, esta pesquisa não contemplou estudos relacionados ao desenvolvimento de software livre.

Outro ponto importante é que também não foram considerados os estudos relacionados a sites de crowdfunding, ou seja, sites onde é realizado o financiamento coletivo de projetos, como é o caso do Lánzanos, citado anteriormente. Apesar de a definição de Estellés-Arolas e Guevara (2012) classificar esta prática como crowdsourcing, a participação nesse tipo de atividade é completamente diferente da participação em outras modalidades de crowdsourcing. Enquanto as demais modalidades em geral demandam tempo e alguma espécie de dedicação por parte dos participantes, no caso do crowdfunding a participação resume-se a investir uma quantia monetária em determinado projeto. Essa diferença na essência da atividade do participante pode resultar em motivações completamente distintas. Isso justifica não terem sido analisados os estudos envolvendo patrocínio coletivo de projetos.

Sundic e Leitner (2013) classificam os projetos de crowdsourcing em plataformas de resolução de problemas e plataformas de geração de ideias. O primeiro tipo focaria na realização de tarefas ou na resolução de problemas específicos, enquanto o segundo tipo focaria na exploração da criatividade da multidão. Porém, considerando a classificação que aparece em Varshney (2012), foram identificadas quatro modalidades distintas de crowdsourcing.

Para este estudo será considerada a classificação de Varshney (2012), apresentada na Tabela 2:

\section{Tabela 2}

Modalidades de Crowdsourcing

\begin{tabular}{c|l|c}
\hline Modalidade & \multicolumn{1}{c|}{ Descrição } & Exemplos \\
\hline $\begin{array}{c}\text { Plataformas } \\
\text { de micro } \\
\text { tarefas } \\
\text { (PMT) }\end{array}$ & $\begin{array}{l}\text { Plataformas onde o crowdsourcer em geral oferece } \\
\text { pequenas remunerações em troca da realização de tarefas } \\
\text { simples como, por exemplo, a categorização de imagens, } \\
\text { transcrição de áudio, traduções, etc. }\end{array}$ & $\begin{array}{c}\text { hwww.mturk.com } \\
\text { http://www.crowdflower.com }\end{array}$ \\
\hline $\begin{array}{c}\text { Concursos, } \\
\text { com ou sem } \\
\text { votação dos } \\
\text { pares } \\
\text { (CON) }\end{array}$ & $\begin{array}{l}\text { Iniciativas onde o crowdsourcer solicita a resolução de um } \\
\text { problema específico e oferece uma recompensa para } \\
\text { aqueles que apresentarem as melhores soluções. A escolha } \\
\text { dos vencedores pode ser feita tanto pelo próprio } \\
\text { crowdsourcer quanto pela multidão por meio de } \\
\text { mecanismos de votação. Entram nessa categoria os } \\
\text { concursos para resolução de problemas complexos, como } \\
\text { problemas de pesquisa e desenvolvimento, além de } \\
\text { concursos para geração de ideias (Idea Contests). }\end{array}$ & \\
\hline
\end{tabular}




\begin{tabular}{|c|c|c|}
\hline $\begin{array}{l}\text { Equipe de } \\
\text { trabalho } \\
\text { colaborativa } \\
\text { (ETC) }\end{array}$ & $\begin{array}{l}\text { São iniciativas que utilizam o esforço colaborativo conjunto } \\
\text { de um grande número de indivíduos para realizar tarefas ou } \\
\text { resolver problemas. Normalmente, problemas mais } \\
\text { complexos são desmembrados em atividades mais simples } \\
\text { que podem ser delegadas à multidão. Nesta categoria entra, } \\
\text { por exemplo, a terceirização de atividades de pesquisa para } \\
\text { a população em geral, conceito conhecido como } \\
\text { crowdscience. }\end{array}$ & $\begin{array}{l}\text { https://www.zooniverse.org } \\
\text { stardustathome.ssl.berkeley.edu }\end{array}$ \\
\hline $\begin{array}{l}\text { Mercados } \\
\text { Preditivos } \\
\quad \text { (MP) }\end{array}$ & $\begin{array}{l}\text { São plataformas eletrônicas em que é possível negociar } \\
\text { títulos cujo retorno está atrelado ao resultado de eventos } \\
\text { futuros. A ideia é que o mecanismo de mercado agregará no } \\
\text { preço dos títulos toda a informação que os participantes } \\
\text { possuem sobre o respectivo evento. Assim, esta } \\
\text { modalidade inclui as iniciativas que visam a gerar previsões } \\
\text { ou monitorar eventos utilizando o mecanismo de mercado } \\
\text { para agregar o conhecimento coletivo. }\end{array}$ & $\frac{\text { http://www.hsx.com }}{\text { http://home.inklingmarkets.com }}$ \\
\hline
\end{tabular}

Fonte: Elaborado pelos autores, com base em Varshney (2012).

Porém, independente da modalidade desenvolvida, a teoria aponta que o sucesso de ações de crowdsourcing depende em grande parte do engajamento das pessoas (Malone et al.., 2010). Apesar disso, a questão de como fomentar a participação em iniciativas de crowdsourcing ainda permanece sem uma resposta satisfatória (Brabham, 2008a).

Apesar de possivelmente existirem motivações que ajudam a explicar a participação em qualquer contexto de crowdsourcing, cada iniciativa apresenta particularidades que podem influenciar a relevância de cada fator (Antikainen, Mäkipää, \& Ahonen, 2010; Brabham, 2010; Kaufmann et al.., 2011). Como modalidades distintas de crowdsourcing compartilham características comuns, é possível que os fatores mais importantes para a participação das pessoas variem em função da respectiva modalidade. A partir deste raciocínio, elaborou-se a hipótese desta pesquisa:

- H1: Existe diferença entre os fatores mais relevantes para a participação das pessoas em modalidades distintas de crowdsourcing.

\section{Metodologia}

Os dados analisados nesta pesquisa foram obtidos em outros estudos acadêmicos. Por meio de uma extensa revisão da bibliografia existente sobre 
motivações para participação em projetos de crowdsourcing, foi possível atingir os objetivos propostos e testar a hipótese de pesquisa.

Para mapear a literatura sobre o tema, foram realizadas buscas em quatro dos principais repositórios de bases de dados de artigos acadêmicos: Web of Science (todas as bases de dados disponíveis), EBSCO (Business Source Complete), Proquest ( $A B I / I N F O R M$ Complete) e Google Acadêmico.

As buscas foram feitas nos principais campos e envolveram termos como "crowdsourcing", "prediction markets" e "idea markets", associados a termos relacionados à participação como, por exemplo, "participation", "motivation", "motive", "intention", etc. Foram utilizados termos em inglês e português.

Em alguns casos, as buscas retornaram muitos resultados e foi necessário um filtro inicial. Por exemplo, uma das buscas no Proquest retornou mais de 1600 registros e neste caso o filtro inicial consistiu em manter apenas artigos com texto completo, em inglês ou português, e cujo campo assunto indicava termos relacionados ao interesse da pesquisa (crowdsourcing/motivações).

O próximo passo foi realizar uma análise dos títulos e resumos dos artigos restantes. Considerando todas as bases de dados consultadas, no total foram analisados os títulos e/ou resumos de mais de 500 trabalhos. Além de textos que evidentemente não estavam relacionados ao tema de interesse e dos trabalhos duplicados, foram descartadas também pesquisas relacionadas à participação em projetos de Open Source e crowdfunding, conforme explicado anteriormente.

Os trabalhos restantes ao final desse processo foram verificados em profundidade para descobrir se o estudo investigava ao menos um fator que poderia influenciar a participação em projetos de crowdsourcing. Nas referências bibliográficas desses trabalhos foram identificados outros estudos relevantes que também foram consultados. No total, essa etapa envolveu a análise detalhada de 84 trabalhos dos quais apenas 36 de fato traziam algum possível motivador para a participação.

Esses 36 trabalhos selecionados foram os efetivamente utilizados nas análises apresentadas a seguir. Nem todos possuem como objetivo principal de pesquisa o estudo da participação. Todavia, em cada um dos estudos selecionados foi identificado algum fator relevante a partir da análise de dados obtidos em um ou mais projetos de crowdsourcing. As características dos estudos são bastante 
diversas: analisam diferentes modalidades, possuem diferentes níveis de rigor metodológico e, enquanto alguns utilizam abordagens quantitativas, outros focam na análise de dados qualitativos obtidos, por exemplo, por meio de entrevistas com usuários. Pesquisas exclusivamente bibliográficas foram descartadas nas etapas anteriores.

Os fatores relevantes para a participação, identificados em cada um dos trabalhos, foram mapeados e tabulados de modo a gerar uma visão consolidada. Como nem sempre os autores utilizam os mesmos termos para se referirem a conceitos semelhantes, e também para reduzir o número total de fatores, itens equivalentes ou semelhantes foram combinados de modo a representarem um único fator. Foram selecionados, então, os fatores que apareceram em seis ou mais trabalhos, ou seja, os fatores que estavam presentes em, no mínimo, 17\% dos trabalhos selecionados.

\section{Apresentação e Discussão dos Resultados}

Uma visão consolidada dos resultados dos 36 estudos selecionados é apresentada na Tabela 3. Além de comparar os fatores identificados em cada um dos trabalhos avaliados, essa tabela também apresenta a modalidade de crowdsourcing que cada estudo investigou, seguindo definições apresentadas anteriormente na Tabela 2.

\section{Tabela 3}

Fatores que Influenciam a Participação em Iniciativas de Crowdsourcing

\begin{tabular}{|c|c|c|c|c|c|c|c|c|c|c|c|c|c|c|c|}
\hline & & $+\operatorname{lr}$ & trín & seca & & & Mo & ivaç & ões & & & + Ex & xtríns & eca & \\
\hline Autores & 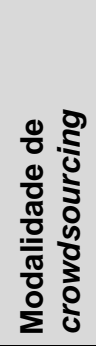 & 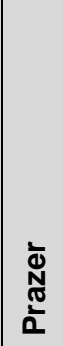 & 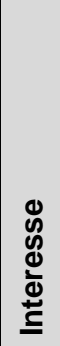 & 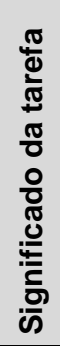 & $\begin{array}{l}\frac{\pi}{\underline{E}} \\
\overline{0} \\
\frac{0}{0} \\
\frac{0}{3} \\
\frac{\mathcal{L}}{4}\end{array}$ & 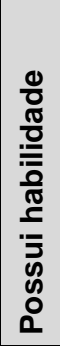 & 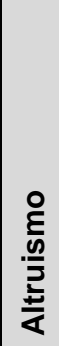 & 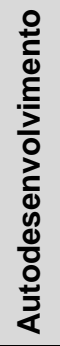 & $\begin{array}{l}\overline{\frac{\pi}{0}} \\
\text { க } \\
\text { ch }\end{array}$ & 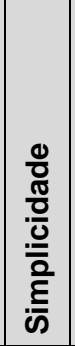 & 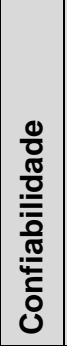 & 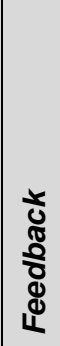 & 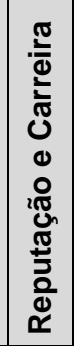 & 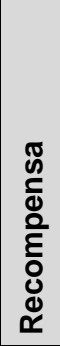 & $\begin{array}{l}\text { : } \\
\text { 올 } \\
\text { ठే }\end{array}$ \\
\hline Chandler; Kapelner, 2013 & PMT & & & $\mathrm{X}$ & & & & & & & & & & & \\
\hline Kaufmann et al.., 2011 & PMT & $\mathrm{X}$ & & $\mathrm{X}$ & $\mathrm{X}$ & $\mathrm{X}$ & & $\mathrm{X}$ & $\mathrm{X}$ & & & $\mathrm{X}$ & $\mathrm{X}$ & $\mathrm{X}$ & $\mathrm{X}$ \\
\hline Kazai et al.., 2013 & PMT & $\mathrm{X}$ & & & & & $\mathrm{X}$ & & & & & & $\mathrm{X}$ & $\mathrm{X}$ & \\
\hline Pilz; Gewald, 2013 & PMT & $\mathrm{X}$ & & $\mathrm{X}$ & $\mathrm{X}$ & $\mathrm{X}$ & & $\mathrm{X}$ & $\mathrm{X}$ & & & $\mathrm{X}$ & $\mathrm{X}$ & $\mathrm{X}$ & $\mathrm{X}$ \\
\hline Rogstadius et al.., 2011 & PMT & & & & & & & & & & & & & $\mathrm{X}$ & \\
\hline Villarroel; Tucci, 2010 & PMT & $\mathrm{X}$ & & & & & & & & & & & $\mathrm{X}$ & $x$ & $\mathrm{X}$ \\
\hline Antikainen et al..,, 2010 & CON & $\mathrm{X}$ & & $\mathrm{X}$ & & & & $\mathrm{X}$ & $\mathrm{X}$ & $\mathrm{X}$ & & $\mathrm{X}$ & & $\mathrm{X}$ & $X$ \\
\hline
\end{tabular}

@(@) Revista Gestão \& Tecnologia, Pedro Leopoldo, v. 17, n. 1, p. 107-129, jan./abr. 2017118 


\begin{tabular}{|c|c|c|c|c|c|c|c|c|c|c|c|c|c|c|c|}
\hline Brabham, 2010 & CON & & & & & & & $\mathrm{X}$ & $\mathrm{X}$ & & & & $\mathrm{X}$ & $\mathrm{X}$ & $\mathrm{x}$ \\
\hline Brabham, 2012 & CON & $\mathrm{X}$ & & & & & $\mathrm{X}$ & $\mathrm{X}$ & & $\mathrm{X}$ & & & $\mathrm{X}$ & & $\mathrm{X}$ \\
\hline Chen et al.., 2012 & CON & & & & & & & & $\mathrm{X}$ & & & $\mathrm{X}$ & & & \\
\hline Fuller, 2006 & $\mathrm{CON}$ & $\mathrm{X}$ & & & & & & & & & & & & $x$ & $x$ \\
\hline Linkruus et al..,, 2012 & $\mathrm{CON}$ & $x$ & & & & & & & $\mathrm{X}$ & & & & & $x$ & \\
\hline Kosonen et al., 2012 & CON & & & & & & $x$ & $\mathrm{X}$ & $\mathrm{X}$ & & & & $\mathrm{X}$ & $\mathrm{X}$ & \\
\hline Kosonen et al., 2013 & CON & & & & & & & & & $X$ & $X$ & & & & $\mathrm{X}$ \\
\hline Leimeister et al., 2009 & $\mathrm{CON}$ & & & & & & & $\mathrm{x}$ & $\mathrm{X}$ & & & & $\mathrm{x}$ & $x$ & \\
\hline Muhdi; Boutellier, 2011 & CON & $\mathrm{X}$ & $\mathrm{X}$ & & & & & $\mathrm{X}$ & & $\mathrm{X}$ & & $\mathrm{X}$ & & $\mathrm{X}$ & $\mathrm{X}$ \\
\hline Shao et al., 2012 & CON & & & & & & & & & $\mathrm{x}$ & & & & $\mathrm{X}$ & $\mathrm{X}$ \\
\hline Son et al., 2012 & $\mathrm{CON}$ & $\mathrm{X}$ & & & & & & & & & & & & & $x$ \\
\hline Sun et al., 2012 & CON & $\mathrm{X}$ & & & & $\mathrm{X}$ & & & & $\mathrm{X}$ & & & & $\mathrm{X}$ & \\
\hline Sundic; Leitner, 2013 & $\mathrm{CON}$ & $\mathrm{X}$ & $X$ & & & & & $x$ & $\mathrm{X}$ & & & & $x$ & $x$ & \\
\hline Tran et al., 2012 & $\mathrm{CON}$ & & & & & & & & & $X$ & & & & & \\
\hline Varshney, 2012 & CON & $\mathrm{X}$ & & & $\mathrm{X}$ & $\mathrm{X}$ & & $\mathrm{X}$ & & & & & $\mathrm{x}$ & $\mathrm{X}$ & \\
\hline Zheng et al., 2011 & CON & $\mathrm{X}$ & & & $\mathrm{X}$ & & & & & $\mathrm{X}$ & $\mathrm{X}$ & & $\mathrm{x}$ & & $\mathrm{x}$ \\
\hline Alam; Campbell, 2012 & ETC & $\mathrm{X}$ & $x$ & $\mathrm{x}$ & $x$ & & $\mathrm{X}$ & $\mathrm{X}$ & & $X$ & $\mathrm{X}$ & $\mathrm{X}$ & $\mathrm{X}$ & & $x$ \\
\hline Brabham, 2008b & ETC & $X$ & & & $X$ & & & $\mathrm{X}$ & $\mathrm{X}$ & & & & $\mathrm{X}$ & $X$ & \\
\hline $\begin{array}{l}\text { Budhathoki; Haythornthwaite, } \\
2013\end{array}$ & ETC & $\mathrm{X}$ & & $\mathrm{x}$ & $\mathrm{X}$ & $\mathrm{x}$ & $\mathrm{x}$ & $\mathrm{X}$ & $x$ & & $\mathrm{X}$ & & & & $\mathrm{x}$ \\
\hline Nov et al., 2011 & ETC & $\mathrm{X}$ & & $\mathrm{x}$ & & & & & & & & & & & $\mathrm{x}$ \\
\hline Nov, 2007 & ETC & $\mathrm{X}$ & & & & & $X$ & $\mathrm{X}$ & & & & & $\mathrm{x}$ & & $x$ \\
\hline Raddick et al., 2010 & ETC & $\mathrm{X}$ & $\mathrm{X}$ & $\mathrm{X}$ & & & $\mathrm{X}$ & $\mathrm{X}$ & $\mathrm{X}$ & & & & & & \\
\hline Sauermann; Franzoni, 2013 & ETC & & $\mathrm{X}$ & & & & & & & & & & & & \\
\hline Vaataja, 2012 & ETC & $X$ & & & & & $\mathrm{X}$ & $\mathrm{X}$ & & & & & & $\mathrm{X}$ & $\mathrm{x}$ \\
\hline Arnesen, 2011 & MP & & & & & & & & & & & & & $\mathrm{X}$ & \\
\hline Montgomery et al., 2013 & MP & $\mathrm{X}$ & & & & $x$ & $\mathrm{X}$ & & & & $\mathrm{X}$ & & $\mathrm{x}$ & $\mathrm{X}$ & $\mathrm{x}$ \\
\hline Gatara, 2012 & Geral & & & & & & & $\mathrm{X}$ & $\mathrm{X}$ & & $\mathrm{X}$ & & $\mathrm{X}$ & & \\
\hline Organisciak, 2010 & Geral & $\mathrm{X}$ & $\mathrm{X}$ & $\mathrm{X}$ & & $\mathrm{X}$ & $x$ & & $\mathrm{X}$ & $\mathrm{X}$ & $\mathrm{X}$ & $x$ & $\mathrm{X}$ & $\mathrm{X}$ & $\mathrm{X}$ \\
\hline Peng Zhang, 2010 & - & & & & & & & $\mathrm{x}$ & $\mathrm{X}$ & & & & $\mathrm{X}$ & $\mathrm{X}$ & \\
\hline Total & & 23 & 6 & 9 & 7 & 7 & 10 & 18 & 15 & 10 & 7 & 7 & 18 & 22 & 19 \\
\hline
\end{tabular}

Fonte: dados da pesquisa.

Foram considerados mais relevantes os fatores que apareceram em um número maior de estudos. Considerando este critério, a Tabela 4 apresenta os treze fatores resultantes por ordem de relevância. São eles: prazer, recompensa, autodesenvolvimento, reputação e carreira, social, altruísmo, simplicidade, significado da tarefa, autonomia, possui habilidade, confiabilidade, feedback e interesse. 
Tabela 4

Relevância dos Fatores que Influenciam a Participação em Crowdsourcing

\begin{tabular}{cccc}
\hline Fator & Classificação & $\begin{array}{c}\text { Qtde de Trabalhos } \\
\text { em que o fator } \\
\text { aparece }\end{array}$ & $\begin{array}{c}\% \text { dos } \\
\text { trabalhos }\end{array}$ \\
\hline Prazer & Intrínseca & 23 & $64 \%$ \\
\hline Recompensa & Extrínseca & 22 & $61 \%$ \\
\hline Autodesenvolvimento & Ambas & 18 & $50 \%$ \\
\hline Reputação e Carreira & Extrínseca & 18 & $50 \%$ \\
\hline Social & Ambas & 15 & $42 \%$ \\
\hline Altruísmo & Intrínseca & 10 & $28 \%$ \\
\hline Simplicidade & Ambas & 10 & $28 \%$ \\
\hline Significado da tarefa & Intrínseca & 9 & $25 \%$ \\
\hline Autonomia & Intrínseca & 7 & $19 \%$ \\
\hline Possui habilidade & Intrínseca & 7 & $19 \%$ \\
\hline Confiabilidade & Ambas & 7 & $19 \%$ \\
\hline Feedback & Extrínseca & 7 & $17 \%$ \\
\hline Interesse & Intrínseca & 6 &
\end{tabular}

Fonte: dados da pesquisa

Baseando-se em classificações propostas por Kaufmann et al. (2011), Brabham (2012) e Budhathoki e Haythornthwaite (2013), cada um dos fatores foi classificado como motivação intrínseca (fruto apenas da realização da própria tarefa) ou extrínseca (oriunda de causas externas à realização da tarefa em si). Quando o fator claramente se encaixava nas duas classificações, ou quando a dúvida persistia mesmo após analisar os trabalhos que identificaram o fator, este foi classificado como "ambas" (Tabela 4).

O primeiro fator em ordem de relevância, denominado "prazer", é autoexplicativo e diz respeito ao nível de prazer que o indivíduo tem ao participar. Há diversas razões que poderiam levar um indivíduo a sentir prazer participando de um projeto de crowdsourcing como, por exemplo, a curiosidade, o desafio percebido, ou mesmo a simples sensação de diversão ao participar.

A "recompensa" representa um prêmio, ou algo que o indivíduo recebe em troca da sua participação. Ela pode estar atrelada ou não ao desempenho do participante e pode variar desde pequenos prêmios até grandes recompensas em dinheiro.

"Autodesenvolvimento" representa o quanto o indivíduo percebe que pode aprender participando da ação de crowdsourcing. Este fator pode ser considerado tanto uma motivação intrínseca quanto extrínseca, pois o desejo de aprender pode 
ser fruto do prazer de ampliar o próprio conhecimento ou pode ter uma razão instrumentalizada como, por exemplo, conseguir um novo emprego.

O fator "reputação e carreira" diz respeito ao quanto o indivíduo percebe que a participação no projeto de crowdsourcing pode representar uma oportunidade para ele fazer uma espécie de marketing pessoal, melhorando sua reputação e ampliando suas possibilidades de carreira. O reconhecimento almejado pode ser tanto dos pares, quanto do patrocinador do projeto.

O fator "social" representa todos os motivos relacionados à interação ou identificação com a comunidade de participantes. Pode significar o desejo de encontrar pessoas com comportamentos e gostos parecidos, fazer novas amizades, ou mesmo significar a pressão social que pode existir para que o indivíduo participe da iniciativa.

O item denominado "altruísmo" diz respeito ao desejo genuíno de ajudar. Faz parte deste fator tanto o desejo de ajudar o mundo em geral, a empresa ou entidade patrocinadora do projeto, ou mesmo ajudar o grupo com o qual o indivíduo se identifica.

"Simplicidade" foi a denominação escolhida para tudo aquilo que torna fácil a experiência de participar do projeto. Baixas barreiras de entrada como a não necessidade de registro ou cadastros extremamente simples, facilidade de uso e telas amigáveis quando a participação exige a interação com um site ou software específico e a proposição de tarefas não muito complexas são todos exemplos de itens que este fator engloba.

"Significado da tarefa" é o quanto a tarefa proposta pelo "crowdsourcer" tem de significado para o indivíduo que está participando. Por exemplo, a simples categorização de imagens quaisquer terá, provavelmente, muito menos significado do que a categorização de imagens que ajudarão a identificar a cura para determinada doença. Este fator representa também o quanto o indivíduo se identifica com a tarefa proposta.

O fator "autonomia" significa o quanto o indivíduo se sente livre para desempenhar a tarefa demandada. Espera-se que quanto maior for a autonomia percebida, maior será a propensão do indivíduo a participar.

"Possui habilidade" representa o fato de que os indivíduos parecem mais propensos a participar de iniciativas em que acreditam possuir as habilidades 
necessárias para o desempenho da tarefa. Uma das razões para esta constatação é que, ao buscar realizar uma tarefa para a qual se sente capaz, o indivíduo está satisfazendo uma necessidade de autoafirmação, mostrando a si mesmo o quanto ele é capaz. Este conceito está relacionado ao termo autoeficácia.

"Confiabilidade", por sua vez, representa diversas dimensões relacionadas à confiança. Por exemplo, a confiança no patrocinador do projeto, no sistema técnico utilizado ou ainda a confiança de que os resultados do projeto serão realmente utilizados. O fator inclui ainda a percepção dos participantes de que o crowdsourcer confia neles.

O fator "feedback" significa que o participante espera que o patrocinador, bem como outros participantes, se interessem por suas contribuições. Desse modo, ele espera receber comentários e avaliações de suas contribuições e, ao satisfazer essa expectativa, ele se sente mais motivado a participar. Este fator está relacionado ao autodesenvolvimento, uma vez que o feedback recebido proporciona aprendizado.

Por fim, o último fator, denominado "interesse", diz respeito ao quanto o indivíduo se interessa pelo assunto tratado no projeto de crowdsourcing. O simples interesse em astronomia já pode ser uma forte razão para o indivíduo participar de um projeto de crowdscience cujo objetivo seja a identificação de novas galáxias, por exemplo. Este item é diferente do fator "significado da tarefa": uma pessoa pode achar importante o desenvolvimento científico, ou seja, a ampliação do conhecimento sobre o universo pode ter um significado para ela, mas é possível que esta pessoa não tenha um interesse particular na tarefa de identificar galáxias.

Com base nos dados da Tabela 3, foi investigado se existe diferença na relevância dos fatores para cada modalidade (conforme divisão de modalidades da Tabela 2). No entanto, Mercados Preditivos (MP) foram apontados por apenas dois dos estudos analisados e, por isso, essa modalidade não foi considerada na análise feita. Da mesma forma, como a análise discutida a seguir envolveu o cálculo de proporções, foi importante filtrar apenas os fatores intrínsecos e extrínsecos mais presentes. Assim, foram considerados apenas os dois mais importantes de cada tipo, a saber: "prazer" / "altruísmo" e "recompensa" / "reputação e carreira".

Verifica-se na Tabela 5 que os fatores extrínsecos são muito mais fortes nas modalidades PMT (Plataforma de Micro Tarefa) e CON (Concursos). O fator "recompensa", por exemplo, aparece em $83 \%$ dos trabalhos que estudam a modalidade PMT e em $65 \%$ dos trabalhos da modalidade CON, valores muito @evista Gestão \& Tecnologia, Pedro Leopoldo, v. 17, n. 1, p. 107-129, jan./abr. 2017122 
superiores aos $25 \%$ dos trabalhos da modalidade ETC (Equipe de Trabalho Colaborativa). O oposto acontece com as motivações intrínsecas: o fator "altruísmo" está presente em apenas $17 \%$ e $12 \%$ das modalidades PMT e CON, respectivamente, mas aparece em $63 \%$ dos trabalhos da modalidade ETC. Estas diferenças são facilmente explicadas: em geral, na modalidade ETC as iniciativas envolvem atividades de grande significado, com apelo colaborativo, como projetos que visam a ajudar pesquisas científicas, por exemplo, e que normalmente não oferecem nenhuma recompensa em dinheiro. Já nas outras duas modalidades, a recompensa financeira é muito mais comum.

O número reduzido de trabalhos em cada categoria, entretanto, impede a realização de testes estatísticos para verificar se essas diferenças são significantes. Para tentar analisar a significância das diferenças entre modalidades, foi necessária uma abordagem um pouco diferente: em vez de verificar diferenças na relevância de cada fator contando quantos trabalhos identificaram cada um, os fatores foram agrupados entre intrínsecos e extrínsecos, conforme classificação apresentada anteriormente (fatores classificados como "ambos" não entraram na análise), e foi feita uma contagem de quantas vezes foram reportados fatores intrínsecos e extrínsecos em cada modalidade.

\section{Tabela 5}

Relevância dos Fatores por Modalidade de Crowdsourcing

\begin{tabular}{cccccc}
\hline & & \multicolumn{4}{c}{ Modalidade de crowdsourcing } \\
\cline { 2 - 6 } & & PMT & CON & ETC & Total \\
\cline { 2 - 6 } Intrínseca de trabalhos & $\mathbf{6}$ & $\mathbf{1 7}$ & $\mathbf{8}$ & $\mathbf{3 1}$ \\
\cline { 2 - 6 } & Prazer & $67 \%$ & $59 \%$ & $\mathbf{8 8 \%}$ & $\mathbf{6 8 \%}$ \\
\hline \multirow{2}{*}{ Extrínseca } & Altruísmo & $17 \%$ & $12 \%$ & $\mathbf{6 3 \%}$ & $\mathbf{2 6 \%}$ \\
\cline { 2 - 6 } & Recompensa & $\mathbf{8 3 \%}$ & $65 \%$ & $25 \%$ & $\mathbf{5 8 \%}$ \\
\cline { 2 - 6 } & $\begin{array}{c}\text { Reputação e } \\
\text { Carreira }\end{array}$ & $\mathbf{6 7 \%}$ & $41 \%$ & $38 \%$ & $\mathbf{4 5 \%}$ \\
\hline
\end{tabular}

Legenda: PMT = Plataforma de Micro Tarefa; CON = Concursos; ETC = Equipe de Trabalho Colaborativa.

Fonte: dados da pesquisa.

Posteriormente, foi realizado um teste Qui-Quadrado para verificar se existe associação entre a modalidade de crowdsourcing e a predominância de motivações intrínsecas ou extrínsecas. O teste mostrou-se significante $(p<0,05)$, o que significa que de fato existe uma diferença nos fatores mais relevantes entre modalidades distintas. 


\section{Tabela 6}

Modalidades de Crowdsourcing Vs. Motivações Intrínsecas e Extrínsecas

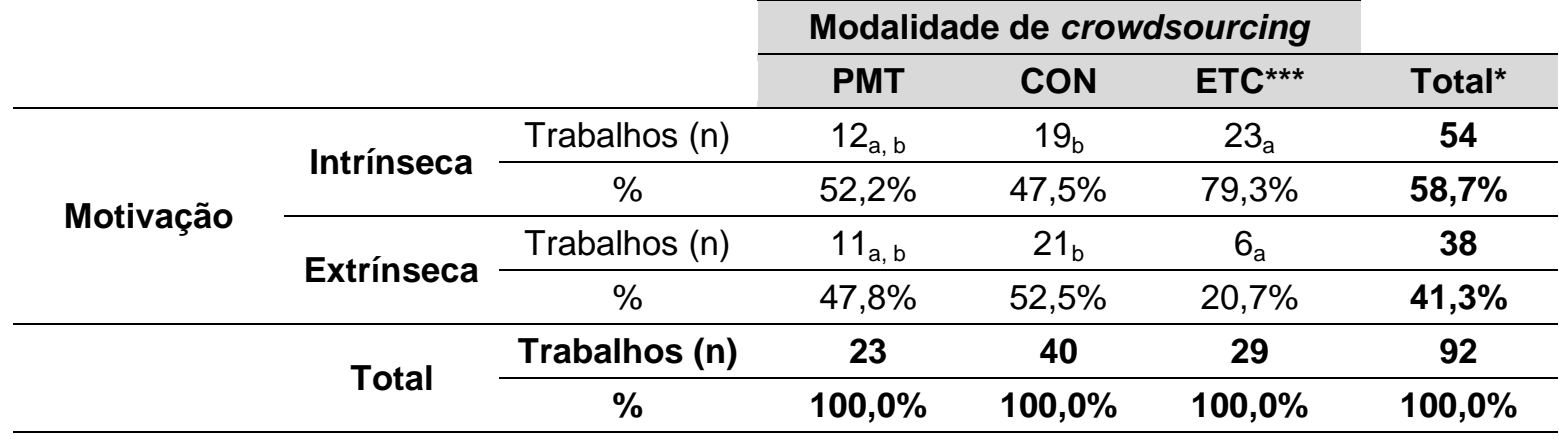

Legenda: $\mathrm{PMT}=$ Plataforma de Micro Tarefa; $\mathrm{CON}=$ Concursos; $\mathrm{ETC}=$ Equipe de Trabalho Colaborativa. Letras subscritas indicam que a proporção daquele tipo de motivação na modalidade não difere significativamente da proporção equivalente em outra modalidade com mesma letra, ao nível de 0,05 . Para cada modalidade foi analisado também se a proporção entre motivações intrínsecas e extrínsecas difere significativamente de um modelo teórico de proporções iguais (50\% intrínsecas - 50\% extrínsecas). Testes significantes são indicados por: * $p<0,1 e^{* * *} p<0,01$.

Analisando a Tabela 6, observa-se que a modalidade ETC é a principal responsável pela diferença identificada. Nesta modalidade, $79,3 \%$ dos fatores são intrínsecos e 20,7\% extrínsecos, diferença significante $(p<0,01)$ quando comparada com um modelo teórico que pressupõe proporções iguais (50\% intrínseco e 50\% extrínseco). Além disso, um teste $Z$ de comparação de proporções mostra uma diferença significante $(p<0,05)$ nas proporções de fatores intrínsecos e extrínsecos entre as modalidades ETC e CON.

Esses achados confirmam a hipótese desta pesquisa $(\mathrm{H} 1)$, ou seja, foram encontradas evidências de que de fato existe diferença entre os fatores mais relevantes para a participação em modalidades distintas de crowdsourcing. Os estudos que analisam a participação em determinada modalidade servem como base, mas não são suficientes para explicar a participação em outras modalidades.

\section{Considerações Finais}

O crowdsourcing pode trazer muitas vantagens, tanto para as empresas (redução de custos, obtenção de soluções inovadoras, etc.) quanto para aqueles que colaboram com essas iniciativas (reconhecimento, aprendizado, etc.). Se bem planejados, os ganhos com projetos de crowdsourcing são muito maiores do que potenciais problemas que podem ocorrer. Para ampliar as chances de sucesso, 
entretanto, sempre será necessário atingir um número adequado de participantes. Portanto, é preciso uma compreensão adequada dos fatores que atraem os usuários.

O primeiro objetivo deste trabalho foi identificar os fatores que influenciam a participação das pessoas em iniciativas de crowdsourcing. A pesquisa levou à identificação de treze fatores relevantes, aqui listados em ordem decrescente de importância: "prazer", "recompensa", "autodesenvolvimento", "reputação e carreira", "social", "altruísmo", "simplicidade", "significado da tarefa", "autonomia", "possui habilidade", "confiabilidade", "feedback" e "interesse".

O segundo objetivo, por sua vez, foi verificar se a relevância dos fatores identificados varia em função de diferentes projetos. Para este objetivo, havia sido definida a hipótese $(\mathrm{H} 1)$ de que existe diferença entre os fatores mais relevantes para a participação das pessoas em modalidades distintas de crowdsourcing. Essa hipótese de pesquisa foi confirmada, uma vez que foi identificada diferença significante entre a proporção de motivações intrínsecas e extrínsecas nas modalidades CON (concursos) e ETC (equipe de trabalho colaborativa).

Esses resultados ampliam o conhecimento existente na literatura sobre crowdsourcing e significam um degrau adicional em direção à consolidação de um modelo que explique adequadamente as relações e forças dos fatores que influenciam a participação. Por outro lado, os resultados sugerem que, devido à diversidade de iniciativas existentes, provavelmente será necessário o desenvolvimento de modelos específicos para projetos de crowdsourcing que compartilhem características comuns.

Os resultados também são relevantes para as organizações interessadas em explorar o poder das multidões. Muitas vezes, preocupados em atrair um grande número de participantes, os patrocinadores desses projetos oferecem prêmios generosos em troca das melhores contribuições. Todavia, este estudo mostrou que em alguns casos essa não é a melhor estratégia, uma vez que outros fatores podem ser ainda mais relevantes do que uma recompensa para alavancar a participação. Satisfazer fatores como "prazer" ou "autodesenvolvimento" pode ser mais barato e trazer melhores retornos. Outra implicação importante para as organizações é que a definição da estratégia para atrair participantes deve considerar as características específicas de cada projeto. 
Uma limitação do presente estudo é o fato de ele que foi baseado em dados secundários obtidos em um número limitado de trabalhos acadêmicos. Esses trabalhos possuem características diversas e variam bastante em termos de rigor e procedimentos metodológicos. Resultados mais robustos talvez possam ser obtidos a partir de pesquisas que levantem os mesmos dados primários e sigam a mesma metodologia de análise para diversas modalidades de crowdsourcing. Esta é exatamente uma das sugestões para estudos futuros sobre o tema. Outra sugestão seria a proposição e teste de um modelo que possa prever o nível de participação em um projeto de determinada modalidade, com base na mensuração dos fatores identificados neste estudo.

\section{Referências}

Alam, S. L., \& Campbell, J. (2012, January). Crowdsourcing motivations in a not-forprofit GLAM context: the Australian newspapers digitisation program. In ACIS 2012: Location, location, location: Proceedings of the 23rd Australasian Conference on Information Systems 2012 (pp. 1-11). ACIS.

Antikainen, M., Mäkipää, M., \& Ahonen, M. (2010). Motivating and supporting collaboration in open innovation. European Journal of Innovation Management, 13(1), 100-119..

Arnesen, S. (2012). Leaping into the unknown: Comparing, testing, and applying methods of predicting elections..

Brabham, D. C. (2008a). Crowdsourcing as a model for problem solving an introduction and cases. Convergence: the international journal of research into new media technologies, 14(1), 75-90.

Brabham, D. C. (2008b). Moving the crowd at iStockphoto: The composition of the crowd and motivations for participation in a crowdsourcing application. First monday, 13(6).

Brabham, D. C. (2010). Moving the crowd at Threadless: Motivations for participation in a crowdsourcing application. Information, Communication \& Society, 13(8), 1122-1145.

Brabham, D. C. (2012). Motivations for participation in a crowdsourcing application to improve public engagement in transit planning. Journal of Applied Communication Research, 40(3), 307-328.

Budhathoki, N. R., \& Haythornthwaite, C. (2013). Motivation for open collaboration crowd and community models and the case of OpenStreetMap. American Behavioral Scientist, 57(5), 548-575. 
Castells, M. (1999). A sociedade em rede (2a ed.). São Paulo: Paz e Terra.

CGI.BR (2016). TIC domicílios 2015: pesquisa sobre o uso das tecnologias de informação e comunicação nos domicílios brasileiros. São Paulo: Comitê Gestor da Internet no Brasil.

Chandler, D., \& Kapelner, A. (2013). Breaking monotony with meaning: Motivation in crowdsourcing markets. Journal of Economic Behavior \& Organization, 90, 123133.

Chen, L., Marsden, J. R., \& Zhang, Z. (2012). Theory and analysis of companysponsored value co-creation. Journal of Management Information Systems, 29(2), 141-172.

Deci, E. L., \& RYAN, R. M. (1985). Intrinsic Motivation and Self-Determination in Human Behavior. Nova York: Plenum Press.

Estellés-Arolas, E., \& González-Ladrón-De-Guevara, F. (2012). Towards an integrated crowdsourcing definition. Journal of Information science, 38(2), 189-200.

Füller, J. (2006). Why consumers engage in virtual new product developments initiated by producers. NA-Advances in Consumer Research Volume 33.

Gatara, M. (2013). Predicting intention to participate in mobile crowdsourcing initiatives: a study of local Kenyan communities (Doctoral dissertation).

Howe, J. (2006). The rise of crowdsourcing. Wired magazine, 14(6), 1-4.

Kaufmann, N., Schulze, T., \& Veit, D. (2011, August). More than fun and money. Worker Motivation in Crowdsourcing-A Study on Mechanical Turk. In AMCIS (Vol. 11, pp. 1-11).

Kazai, G., Kamps, J., \& Milic-Frayling, N. (2013). An analysis of human factors and label accuracy in crowdsourcing relevance judgments. Information retrieval, 16(2), 138-178.

Kosonen, M., Gan, C., Blomqvist, K., \& Vanhala, M. (2012, January). Users' motivations and knowledge sharing in an online innovation community. In ISPIM Conference Proceedings (p. 1). The International Society for Professional Innovation Management (ISPIM).

Kosonen, M., Gan, C., Olander, H., \& Blomqvist, K. (2013). My idea is our idea! Supporting user-driven innovation activities in crowdsourcing communities. International Journal of Innovation Management, 17(03), 1340010.

Leimeister, J. M., Huber, M., Bretschneider, U., \& Krcmar, H. (2009). Leveraging crowdsourcing: activation-supporting components for IT-based ideas competition. Journal of management information systems, 26(1), 197-224.. 
Linkruus, K., Nilsson, K., \& Westerberg, A. (2012). Crowdsourcing-Take on Goliath: Motivating people to participate in Crowdsourcing.

Malone, T. W. et al..(2010). The collective intelligence genome. MIT Sloan Management Review, 51(3), 21-31.

Montgomery, T. A., Stieg, P. M., Cavaretta, M. J., \& Moraal, P. E. (2013, August). Experience from hosting a corporate prediction market: benefits beyond the forecasts. In Proceedings of the 19th ACM SIGKDD international conference on Knowledge discovery and data mining (pp. 1384-1392). ACM.

Muhdi, L., \& Boutellier, R. (2011). Motivational factors affecting participation and contribution of members in two different Swiss innovation communities. International Journal of Innovation Management, 15(03), 543-562..

Nov, O. (2007). What motivates wikipedians?. Communications of the ACM, 50(11), 60-64.

Nov, O., Arazy, O., \& Anderson, D. (2011, February). Dusting for science: motivation and participation of digital citizen science volunteers. In Proceedings of the 2011 iConference (pp. 68-74). ACM.

Organisciak, P. (2010). Why bother? Examining the motivations of users in large-scale crowd-powered online initiatives (Masters dissertation).

Peng, L., \& Zhang, M. (2010, November). An empirical study of social capital in participation in online crowdsourcing. In E-Product E-Service and E-Entertainment (ICEEE), 2010 International Conference (pp. 1-4). IEEE.

Pilz, D., \& Gewald, H. (2013). Does Money Matter? Motivational Factors for Participation in Paid-and Non-Profit-Crowdsourcing Communities. In Wirtschaftsinformatik (p. 37).

Raddick, M. J., Bracey, G., Gay, P. L., Lintott, C. J., Murray, P., Schawinski, K., ... \& Vandenberg, J. (2009). Galaxy zoo: Exploring the motivations of citizen science volunteers. arXiv preprint arXiv:0909.2925.

Rogstadius, J., Kostakos, V., Kittur, A., Smus, B., Laredo, J., \& Vukovic, M. (2011). An assessment of intrinsic and extrinsic motivation on task performance in crowdsourcing markets. ICWSM, 11, 17-21.

Sauermann, H., \& Franzoni, C. (2013). Participation dynamics in crowd-based knowledge production: The scope and sustainability of interest-based motivation. Available at SSRN 2360957.

Shao, B., Shi, L., Xu, B., \& Liu, L. (2012). Factors affecting participation of solvers in crowdsourcing: an empirical study from China. Electronic Markets, 22(2), 73-82. 
Son, J., Sadachar, A., Manchiraju, S., Fiore, A. M., \& Niehm, L. S. (2012). Consumer adoption of online collaborative customer co-design. Journal of Research in Interactive Marketing, 6(3), 180-197.

Sun, Y., Fang, Y., \& Lim, K. H. (2012). Understanding sustained participation in transactional virtual communities. Decision Support Systems, 53(1), 12-22.

Šundić, M., \& Leitner, K. H. (2013). Crowdsourcing as an Innovation Strategy: A Study on Innovation Platforms in Austria and Switzerland. Communications \& Strategies, (89), 55-72.

Tran, A., \& Park, J. (2012). Crowd participation pattern in the phases of a product development process that utilizes crowdsourcing. Industrial Engineering and Management Systems, 11(3), 266-275.

Toffler, A. (1980). A terceira onda (3a ed.). Rio de Janeiro: Record.

Väätäjä, H. (2012, October). Readers' motivations to participate in hyperlocal news content creation. In Proceedings of the 17th ACM international conference on Supporting group work (pp. 309-312). ACM.

Varshney, L. R. (2012, October). Participation in crowd systems. In Communication, Control, and Computing (Allerton), 2012 50th Annual Allerton Conference on (pp. 996-1001). IEEE.

Villarroel Fernandez, J. A., \& Tucci, C. L. (2010). Motivating firm-sponsored ecollective work.

Zheng, H., Li, D., \& Hou, W. (2011). Task design, motivation, and participation in crowdsourcing contests. International Journal of Electronic Commerce, 15(4), 5788. 